\title{
Tasa de Derivación y factores asociados a la Calidad de la Hoja de Derivación en Atención Primaria
}

\author{
Pablo Franquelo Moralesa, David García Mateosa , Pablo Moya Martínez ${ }^{\mathrm{b}}$ Jorge Lema Bartoloméc, \\ Jesús Buendía Bermejo ${ }^{d}$, Sagrario Sáiz Santos ${ }^{d}$
}

aServicio de Urgencias. Hospital Virgen de la Luz. Cuenca.

${ }^{\mathrm{b}}$ Centro de Estudios Socio-Sanitarios. CESS. Cuenca.

\section{'Unidad Docente de} Medicina Familiar y Comunitaria. Cuenca.

'Médico de Familia .Centro de Salud Cuenca II. Cuenca.

\section{Correspondencia:}

Pablo Franquelo Morales.

Servicio de Urgencias.

Hospital Virgen de la Luz, C/ Hermandad de Donantes $n^{\circ} 2$ 16002 Cuenca.

Telf.: 969179900

e-mail: pfranquelo@yahoo.es

Recibido el 24 de junio de 2008.

Aceptado para su publicación el 11 de agosto de 2008.

\section{RESUMEN}

Objetivo. 1) Analizar la relación entre la cantidad y calidad de las hojas de interconsulta (IC) y los factores relacionados con el médico de familia (MF). 2) Conocer su distribución por especialidades.

Diseño. Estudio descriptivo, transversal.

Emplazamiento. Centro de salud urbano de la ciudad de Cuenca.

Sujetos. Analizamos un total de 1.214 derivaciones realizadas a través del programa informático de explotación de datos (TURRIANO) por $7 \mathrm{MF}$, durante el último trimestre del año 2005.

Mediciones principales. Se han analizado retrospectivamente las hojas de IC. Recogimos variables asociadas al MF y asociadas a la propia IC (especialidad a la que va dirigida, fecha y calidad). Se establecieron 3 categorías para valorar la calidad de las IC: "mala" (cumplía al menos uno de los criterios "ausencia de motivo de consulta y anamnesis de la enfermedad actual" y "ausencia de exploración física y exploraciones complementarias"), "aceptable" (presencia simultánea de los dos criterios anteriores) y "buena" (cumplía criterios de "aceptable" más la presencia de al menos uno de los siguientes: antecedentes familiares, antecedentes personales, tratamiento habitual, diagnóstico de sospecha).

Resultados. La calidad de las derivaciones fue mala en un $68,2 \%$, aceptable en un $11,4 \%$, y buena en un $20,3 \%$. Los servicios a los que más se derivó fueron Traumatología 14,7\%, Oftalmología 11,8\%, Ginecología $11 \%$ y Dermatología $10,1 \%$.

La proporción de remisiones buena/aceptable en las especialidades más demandadas fue: Dermatología 66,7\%, Traumatología 29,1\%, Otorrinolaringología 23,3\%, Ginecología 19,5\% y Oftalmología 16,8\%. En cuanto a la calidad aceptable/buena de la interconsulta, la variable formación MIR presentó una diferencia estadísticamente significativa $(p=0,03)$. El $28,6 \%$ tenía formación MIR, el $57,1 \%$ eran profesores y el $57,1 \%$ hombres.

Conclusiones. El número de IC realizadas por un equipo de Atención Primaria (EAP) a Atención Especializada $(\mathrm{AE})$ es una referencia de su correcto funcionamiento y de su capacidad de resolución. Encontramos una gran variabilidad en el número de las IC entre los distintos médicos, que no parece justificarse por el número de tarjetas y el volumen de trabajo. Disponer de formación especializada y tutorizar residentes parece relacionarse con mayor capacidad de cumplimentación y calidad de las interconsultas.

Palabras clave. Remisión y Consulta. Atención Primaria de Salud.

\section{ABSTRACT}

Influence of the primary care physician in the quantity and quality of referrals to Specialist Services

Objective. To analyze the relationship between the quantity and quality of referrals and factors related to the family physician (FP). To determine their distribution by specialities.

Design. Descriptive, cross study.

Setting. Primary care centre in Cuenca city.

Subjects. We analyzed a total of 1,214 referrals, using the software package (TURRIANO), made by 7 family physicians during the last quarter of 2005.

Main measurements. Referral forms were analysed retrospectively. We compiled variables associated with the family physician (figure 1) and with the referral itself (speciality to which it is addressed, date and quality).

Three categories were established to assess the quality of referrals. Poor quality referrals met at least one of the 2 following criteria "lack of consultation reason (MC) and current history of the disease (AD)" and "lack of physical (PE) and complementary tests (CT)". In acceptable referrals, the two previous criteria were present simultaneously. Finally, a referral was evaluated as good if it fulfilled all the criteria for being acceptable plus the presence of at least one of the following: Family history, personal history, routine treatment, diagnosis.

Results. We analyzed 1,214 referrals. The quality of the referrals was poor in $68.2 \%$, acceptable in $11.4 \%$ and good in $20.3 \%$. The services that most referrals were made to were Traumatology $14.7 \%$, Ophthalmology $11.8 \%$, Gyneacology $11 \%$, and Dermatology $10.1 \%$. The proportion of good/acceptable referrals in the most popular specialities were: Dermatology $66.7 \%$, Traumatology $29.1 \%$, Otolaryngology $23.3 \%$, Gyneacology $19.5 \%$ and Ophthalmology $16,8 \%$.

With regard to the acceptable /good quality of the referral only the variable training (MIR) presented a statistically significant difference $(p=0,03)$. Of the doctors considered, $28.6 \%$ had MIR training, $57.1 \%$ were tutors and $57.1 \%$ were male.

Conclusions. The number of referrals made by a Primary Health Care team (PC) to Specialist care (SC) is related to correct performance of this team and their ability to solve problems in their daily work.

In our study there was a great variability in the number of referrals made by different doctors, which does not appear to be justified by the number of cards and workload. Having specialist training and tutoring residents appears to be associated with a greater capacity and quality completion of referrals.

Key words. Referral and Consultation. Primary Health Care.

Presentado parcialmente en el VIII Congreso de Atención Primaria de Castilla La Mancha, Albacete junio de 2007, y en el XXVII Congreso SemFYC Valladolid-Salamanca, noviembre del 2007.

Primer premio en la categoría de residente en III Premios de Investigación de Atención Primaria, Cuenca, diciembre del 2007. 


\section{INTRODUCCIÓN}

La Atención Primaria (AP) es el primer punto de contacto de los pacientes con el sistema sanitario. Es objetivo de la misma ofrecer una atención global, integrada, continuadaa y personalizada a los pacientes ${ }^{1-3}$. La mayoría de las consultas se resuelven en AP, pero aproximadamente un $5 \%$ de las mismas se derivan a atención especializada (AE) hospitalaria ${ }^{3-6}$.

A través de la hoja de derivación (HD) se lleva a cabo la comunicación entre AP y AE, fundamental para proporcionar un nivel aceptable de calidad en la práctica clínica. A pesar de que existen distintos factores que influyen en la calidad y la cantidad de las HD, la relevancia de cada uno de ellos no esta suficientemente estudiada $^{7-9}$.

La progresiva informatización de los servicios sanitarios constituye una herramienta de extraordinaria utilidad en la comunicación entre los distintos niveles asistenciales. Para ello, el Servicio de Salud de Castilla-La Mancha (SESCAM) ha diseñado la plataforma informática denominada TURRIANO ${ }^{10}$. Incluye una hoja de derivación en la que se registran Motivo de consulta, Exploración, Aproximación diagnóstica y Plan terapéutico (MEAP).

Son numerosos los estudios sobre derivaciones desde $A P$ a $A E 3,{ }^{11-15}$, pero son muy pocos los que están basados en HD informatizadas ${ }^{15}$.

El objetivo del presente estudio es conocer la tasa de derivación (TD) de un centro de salud de la capital de Cuenca y evaluar los factores que influyen en la cantidad y distribución de las derivaciones a $\mathrm{AE}$, así como la calidad de la hoja de derivación.

\section{MATERIAL Y MÉTODO}

Se desarrolló un estudio observacional transversal en un centro de salud (CS) urbano de la provincia de Cuenca. El equipo del CS constaba de 10 médicos, de los cuales 1 rechazó participar y 2 se excluyeron por asistir únicamente cupos de población infantil. Los 7 que aceptaron lo hicieron voluntariamente. La población de estudio estuvo constituida por todos los pacientes derivados a consultas especializadas y atendidos por dichos médicos durante el periodo de octubre a diciembre de 2005.

Se recogieron las variables asociadas al médico: edad, sexo, años de ejercicio profesional, ser tutor de médicos residentes, tener formación MIR, el tamaño del cupo, la presión asistencial y el número de consultas realizadas durante el periodo de estudio. La presión asistencial se calculó como número de pacientes por profesional y día.

De los pacientes derivados se recogieron: sexo, edad, especialidad a la que fueron derivados, fecha de derivación y una serie de ítems de la hoja de derivación del paciente. Dicha hoja debía ser cumplimentada por los médicos de cada paciente mediante la plataforma informática del SESCAM (TURRIANO), diseñada para la explotación de datos y la gestión administrativa y clínica. Los ítems correspondieron a sí el médico había rellenado o no los siguientes apartados en la HD: motivo de consulta (MC), anamnesis de la enfermedad actual (EA), exploración física (EF), exploraciones complementarias (EC), antecedentes familiares (AF), antecedentes personales (APS), tratamiento habitual (TTO) y diagnóstico de sospecha (DX). Con ellos, y basándonos en la literatura ${ }^{3,5,12,15}$, se clasificaron las $\mathrm{HD}$ en 3 niveles de calidad: deficiente, aceptable y buena (tabla 1).

Además, se obtuvo el número de derivaciones realizadas en cada uno de los CS de la ciudad de Cuenca durante el mismo periodo de estudio.

En cuanto al análisis estadístico, para las variables cuantitativas se calculó la media \pm desviación estándar (DE) y para las cualitativas la proporción e intervalo de confianza (IC) del 95\%. Para encontrar diferencias entre los distintos grupos se empleó la prueba T de Student y Chi-cuadrado de Pearson, en función de la naturaleza de las variables.

Se desarrolló un análisis de regresión logística por pasos. En el primer paso, mediante el método "enter", se incluyeron las variables independientes edad y sexo del paciente, así como la variable médico, y en el segundo, mediante el método "stepwise", cada una de las especialidades a las que se deriva, codificadas como variables de tipo dummy (cero o uno). El objetivo de este análisis fue conocer si un grupo de especialidades permitía explicar la variabilidad en la calidad de las hojas de derivación, realizando un control por las variables confusoras. La variable dependiente fue la calidad de la hoja de derivación en 2 categorías: aceptable-buena o deficiente. Para el análisis estadístico se han empleado los programas SPSS 14.0 y Epidat ${ }^{3.1}$.

\section{RESULTADOS}

Se atendieron 17.441 pacientes, de los cuales 1.161 fueron derivados por primera vez, lo que supuso una tasa de derivación (TD) de 6,66\% (IC 95\%: 6,28-7,05). El $59,9 \%$ de las derivaciones correspondió a mujeres. Por grupos de edad, el 46,3\% fueron menores de 45 años, el $26,0 \%$ población de entre 45 y 65 años y el 
27,6\% fueron mayores de 65 años. La TD por médico osciló entre el $4,38 \%$ y el $10,47 \%$.

La edad media de los médicos fue de 48,86 $\pm 3,23$ DE. De ellos, 4 fueron mujeres y 3 hombres. Sólo 2 tenían formación MIR y además fueron mujeres. Cuatro estaban acreditados como tutores de residentes. La media de años de ejercicio profesional fue de 11,01 \pm 4,75 . El tamaño medio del cupo de los médicos fue de $1.873,14$, con un rango de 1.470-2.144. Los 3 médicos varones realizaron el $50,8 \%$ de las derivaciones, además 2 de ellos derivaron a pacientes de menor edad $(44,42 \pm 18,16$ DE vs $50,85 \pm 19,90 \mathrm{DE} ; \mathrm{p}<0,001)$. La media de presión asistencial fue de 41,11 $\pm 10,01 \mathrm{DE}$. Durante los tres meses de estudio se realizaró una media de 165,85 \pm 76,21 DE derivaciones por médico frente a las $143,76 \pm 50,51 \mathrm{DE}$ y $111,62 \pm 54,73 \mathrm{DE}$ realizadas en los otros 2 CS de la ciudad.

Las derivaciones fueron dirigidas a 27 especialidades. En la tabla 2 se muestra la frecuencia y el porcentaje de derivaciones, así como la edad y el sexo de los pacientes. La especialidad a la que más se derivó fue traumatología (14,72\%), seguida de oftalmología $(11,88 \%)$ y ginecología $(10,59 \%)$. A las que menos se derivó fueron las unidades del dolor $(0,08 \%)$ y del tabaco $(0,25 \%)$, planificación familiar $(0,25 \%)$ y cirugía vascular (0,25\%). En 6 especialidades se agruparon el $60,98 \%$ de las derivaciones.

El porcentaje en que se cumplimentaron los ítems que forman la escala que evalúa la calidad de las HD se muestra en la figura 2. El mayor porcentaje se obtuvo en el ítem motivo de consulta $(97,33 \%$ ) y el menor en antecedentes familiares $(0,78 \%)$. La media en el porcentaje cumplimentado fue de 35,19 $\pm 16,65 \mathrm{DE}$. Se obtuvieron diferencias significativas entre la media del porcentaje cumplimentado para hombres $(36,53$ $\pm 16,69 \mathrm{DE})$ y mujeres $(34,30 \pm 34,30 \mathrm{DE})$, pero cuando controlamos por médico sólo aparecieron en 2 de ellos. En cuanto a la media del porcentaje cumplimentado por edad del paciente no obtuvimos diferencias significativas.

La calidad de las hojas de derivación fue deficiente en el $67^{\prime} 9 \%$ de los casos, aceptable en el $11,6 \%$ y buena en el $20,5 \%$. Existieron diferencias entre las HD aceptables-buenas por especialidad $(p<0,001)$, situándose los mayores porcentajes de estas en las especialidades de urgencias hospitalarias (100\%), dermatología $(66,95 \%)$, medicina interna $(60 \%)$ y cirugía general $(55,41 \%)$. En cuanto al sexo del paciente, no encontramos diferencias con las HD aceptables-buenas $(p=$ 0,061 ), aunque sí que hubo un médico (sin formación MIR y no tutor) con un porcentaje significativamente inferior en calidad aceptable-buena de la HD realizada a mujeres (mujeres: 13,4\%; hombres: 31,1\%; $p=$ $0,01)$. No hubo diferencias en la edad de los pacientes entre las hojas aceptables-buenas y deficientes $(p=$ $0,984)$.

$\mathrm{Ni}$ entre los días laborables de la semana, ni entre los meses (octubre, noviembre y diciembre) hubo diferencias significativas en la calidad de las HD. Cuando controlamos por médico, dos de ellos, varones, sin formación MIR y no tutores, tuvieron un porcentaje significativamente superior de valoraciones aceptables-buenas en el mes de diciembre $(37,5 \%$ y $36,2 \%)$ que en los meses anteriores $(0 \% / 22 \%$ y $12 \% / 15 \%$, respectivamente).

Entre los médicos encontramos diferencias en la calidad de las hojas de derivación $(p<0,001)$. Los médicos mujeres realizaron las hojas con calidad aceptable-buena en un $43,1 \%$ y los hombres en un $21,5 \%$ $(p<0,001)$. Los médicos con formación MIR fueron mujeres y obtuvieron un porcentaje significativamente superior de calidad aceptable-buena $(61,0 \%$ vs $23,4 \% ; p<0,001)$. Entre los que no tuvieron formación MIR no hubo diferencias (hombres: 21,5\%; mujeres: $27,2 \% ; p=0,06$ ). Sí que hubo entre los médicos que

DEFICIENTE, sí ausencia de uno de los dos criterios siguientes:

Motivo de consulta (MC) y anamnesis de la enfermedad actual (EA)

Exploración física (EF) y exploraciones complementarias (EC)

ACEPTABLE, sí presencia de los dos criterios siguientes:

Motivo de consulta (MC) y anamnesis de la enfermedad actual (EA)

Exploración física (EF) y exploraciones complementarias (EC)

BUENA, sí cumple los criterios de ACEPTABLE y presenta alguno de los siguientes:

Antecedentes familiares (AF)

Antecedentes personales (APS)

Tratamiento habitual (TTO)

Diagnóstico de sospecha (Dx)

Tabla 1. Niveles de calidad de la hoja de derivación (Modificada de Rodríguez Alcalá FJ, et al.) 


\begin{tabular}{|c|c|c|c|c|}
\hline Especialidad & Frecuencia & $\begin{array}{l}\text { Porcentaje } \\
\text { ( IC del 95\%) }\end{array}$ & $\begin{array}{c}\text { Edad } \\
\text { (Media } \pm \text { DE) }\end{array}$ & $\begin{array}{c}\text { Mujeres } \\
\%\end{array}$ \\
\hline Traumatología & 171 & $14,72(12,64-16,81)$ & $48,79 \pm 18,85$ & 57,90 \\
\hline Oftalmología & 138 & $11,88(9,98-13,79)$ & $58,50 \pm 20,39$ & 46,40 \\
\hline Ginecología & 123 & $0,59(8,78-12,40)$ & $38,45 \pm 13,44$ & 100,00 \\
\hline Dermatología & 118 & $10,16(8,38-11,94)$ & $49,16 \pm 21,31$ & 53,40 \\
\hline ORL & 84 & $7,23(5,70-8,76)$ & $51,73 \pm 20,58$ & 59,50 \\
\hline Cirugía general & 74 & $6,37(4,92-7,82)$ & $48,78 \pm 19,20$ & 50,00 \\
\hline Digestivo & 65 & $5,59(4,23-6,96)$ & $48,67 \pm 21,78$ & 46,00 \\
\hline Neurología & 48 & $4,13(2,94-5,32)$ & $56,16 \pm 23,01$ & 68,80 \\
\hline Salud mental & 47 & $4,04(2,87-5,22)$ & $38,65 \pm 16,02$ & 61,70 \\
\hline Urología & 44 & $3,78(2,64-4,93)$ & $53,72 \pm 19,02$ & 18,20 \\
\hline Alergología & 40 & $3,44(2,35-4,53)$ & $37,47 \pm 15,43$ & 75,00 \\
\hline Rehabilitación & 35 & $3,01(1,98-4,94)$ & $50,05 \pm 17,23$ & 57,10 \\
\hline Reumatología & 35 & $3,01(1,98-4,94)$ & $55,02 \pm 15,19$ & 74,30 \\
\hline Neumología & 24 & $2,06(1,20-2,92)$ & $50,41 \pm 16,03$ & 33,30 \\
\hline Cardiología & 23 & $1,98(1,13-2,82)$ & $54,08 \pm 19,29$ & 47,80 \\
\hline Endocrinología & 20 & $1,72(0,93-2,51)$ & $50,55 \pm 18,48$ & 60,00 \\
\hline Unidad de mama & 17 & $1,46(0,73-2,19)$ & $46,17 \pm 17,49$ & 94,10 \\
\hline Nefrología & 15 & $1,29(0,59-1,98)$ & $63,00 \pm 9,29$ & 66,70 \\
\hline Geriatría & 11 & $0,94(0,34-1,54)$ & $76,54 \pm 11,63$ & 100,00 \\
\hline UH & 10 & $0,86(0,28-1,43)$ & $53,80 \pm 11,52$ & 40,00 \\
\hline Medicina Interna & 5 & $0,43(0,14-1,00)$ & $69,20 \pm 11,45$ & 60,00 \\
\hline Hematología & 4 & $0,34(0,09-0,88)$ & $11,50 \pm 17,01$ & 25,00 \\
\hline Cirugía vascular & 3 & $0,25(0,05-0,75)$ & $49,33 \pm 8,32$ & 100,00 \\
\hline Planificación familiar & 3 & $0,25(0,05-0,75)$ & $34,66 \pm 10,50$ & 100,00 \\
\hline Unidad de tabaco & 3 & $0,25(0,05-0,75)$ & $52,66 \pm 12,01$ & 33,33 \\
\hline Unidad del dolor & 1 & $0,08(0,00-0,47)$ & 69,00 & 100,00 \\
\hline
\end{tabular}

Tabla 2. Análisis descriptivo de las Derivaciones por especialidad. IC: intervalo de confianza; ORL: otorrinolaringología; UH: urgencias hospitalarias.

eran tutores y los que no $(38,6 \%$ vs $25,0 \% ; p<0,001)$ y respecto a los tutores también existieron diferencias entre los hombres y las mujeres $(21 \%$ vs $61 \%$; $\mathrm{p}<0,001)$

Finalmente, en el análisis de regresión logística con la variable dependiente calidad de la HD (aceptable-buena o mala) 8 especialidades quedaron en el modelo final, indicando que las HD realizadas a las especialidades de cirugía general y digestivo tenían una menor probabilidad de que su calidad fuera deficiente, mientras que las HD de las especialidades de alergología, neumología, ORL, salud mental, rehabilitación y urología aumentaban esta probabilidad (tabla 4).

\section{DISCUSIÓN}

Encontramos dificultades metodológicas para clasificar la calidad de las HD. Los estudios previos utilizaban un modelo de medición basado en papel, sin el empleo de programas informáticos. Estos solucionan los problemas de ilegibilidad, subjetividad y datos administrativos del paciente y del médico, que aparecen en estudios previos como criterios de calidad ${ }^{6,12,16}$. Por este motivo, optamos por utilizar una clasificación propia fundamentada en la presencia o no de ciertos apartados en el cuerpo de la HD, que consideramos básicos en cualquier acto médico.

Las características sociodemográficas y la TD de la población derivada son similares a las de estudios previos $^{2,6,17-18}$. Los médicos varones sin formación MIR derivaron más. Los porcentajes de cumplimentación de la HD revelaron que el motivo de consulta $(97,33 \%)$ y la anamnesis de la enfermedad actual $(55,21 \%)$ son los más registrados, sin embargo las exploraciones complementarias y los antecedentes familiares tan sólo se registraron en un $7,92 \%$ y $0,78 \%$, respectivamente. Esta variabilidad en la cumplimentación puede justificarse porque ciertos apartados como son Antecedentes Familiares o Exploraciones Complementarias no aparecen sistemáticamente y son de difícil registro en la plataforma TURRIANO.

En nuestro estudio obtuvimos unos valores de calidad aceptable-buena que supusieron aproximadamente un tercio del total. Aunque no existen estudios previos 


\begin{tabular}{|c|c|c|c|c|}
\hline \multirow[t]{2}{*}{ Especialidad } & \multicolumn{3}{|c|}{$\begin{array}{c}\text { Calidad } \\
\text { (aceptables-buenas / Total) }\end{array}$} & \multirow[t]{2}{*}{$\begin{array}{c}\text { Total } \\
\text { (\% aceptables-buenas) }\end{array}$} \\
\hline & Mujeres & Hombres & $p$ & \\
\hline Traumatología & $24 / 99$ & $28 / 72$ & 0,059 & 30,41 \\
\hline Oftalmología & $5 / 64$ & $18 / 74$ & 0,018 & 16,67 \\
\hline Ginecología & $25 / 123$ & - & - & 20,33 \\
\hline Dermatología & $42 / 63$ & $37 / 55$ & 0,899 & 66,95 \\
\hline ORL & $11 / 50$ & $8 / 34$ & 0,919 & 22,62 \\
\hline Cirugía general & $19 / 37$ & $22 / 37$ & 0,640 & 55,41 \\
\hline Digestivo & $8 / 30$ & $11 / 35$ & 0,882 & 29,23 \\
\hline Neurología & $7 / 33$ & $0 / 15$ & 0,136 & 14,58 \\
\hline Salud mental & $17 / 29$ & $4 / 18$ & 0,032 & 44,68 \\
\hline Urología & $0 / 8$ & $10 / 36$ & 0,218 & 22,73 \\
\hline Alergología & $2 / 30$ & - & - & 7,50 \\
\hline Rehabilitación & $5 / 20$ & $5 / 15$ & 0,871 & 28,57 \\
\hline Reumatología & $9 / 26$ & $2 / 9$ & 0,784 & 31,43 \\
\hline Neumología & $0 / 8$ & $2 / 16$ & 0,794 & 8,33 \\
\hline Cardiología & $8 / 11$ & $3 / 12$ & 0,061 & 47,83 \\
\hline Endocrinología & $6 / 12$ & $2 / 8$ & 0,514 & 40,00 \\
\hline Unidad de mama & $7 / 16$ & $0 / 1$ & 0,669 & 41,18 \\
\hline Nefrología & $1 / 10$ & $3 / 5$ & 0,148 & 26,67 \\
\hline Geriatría & $5 / 11$ & - & - & 45,45 \\
\hline $\mathrm{UH}$ & $4 / 4$ & $6 / 6$ & - & 100,00 \\
\hline Medicina Interna & $2 / 3$ & $1 / 2$ & - & 60,00 \\
\hline Hematológica & $1 / 1$ & $1 / 3$ & - & 50,00 \\
\hline Cirugía vascular & $1 / 3$ & - & - & 33,33 \\
\hline Planificación familiar & $0 / 3$ & - & - & 0,00 \\
\hline Unidad de tabaco & $0 / 1$ & $0 / 2$ & - & 0,00 \\
\hline Unidad del dolor & $0 / 1$ & - & - & 0,00 \\
\hline Total (\% aceptables-buenas) & $30,0 \%$ & $35,3 \%$ & & 32,1 \\
\hline
\end{tabular}

Tabla 3. Calidad de las hojas de derivación por sexo. ORL: otorrinolaringología; UH: urgencias hospitalarias.

que utilicen la misma metodología y que nos permitan comparar, sí parece que podemos haber empleado unos criterios demasiado estrictos de calidad en la HD.

Al analizar las derivaciones a ciertas especialidades, se objetiva que no precisan cumplir todos los criterios de calidad, ya que en ocasiones no se requiere de Exploración física y/o complementaria, minimizando así la probabilidad de una mala cumplimentación.

Respecto a la calidad en la HD entre especialidades encontramos claras diferencias que se deben sobretodo a la presencia de Exploración física y/o complementarias. No se ha medido la indicación o no de la HD, sobretodo para evitar el componente de subjetividad asociado, aunque sí que consideramos que podría tenerse en cuenta en futuros trabajos sobre el tema.

En la bibliografía revisada sobre la calidad de las HD encontramos resultados muy heterogéneos. Los trabajos que emplean la escala propuesta por Irazábal y
Gutiérrez, modificada de Morera et $\mathrm{al}^{16}$, muestran porcentajes de calidad buena que oscilan entre el 11,5\% y el $61,9 \%$, y de mala calidad entre el $7 \%$ y el $40 \%$, relacionada con la subjetividad a la que se presta este tipo de escalas.

En el análisis de regresión logística se ha constatado una menor probabilidad de cumplimentación deficiente en las HD realizadas a las especialidades de digestivo y cirugía general al controlar por médico, sin ser las más derivadas. Probablemente, el dolor abdominal es uno de los motivos de consulta más frecuentemente registrados y que requiere una mayor precisión en el diagnóstico, recurriendo necesariamente a la exploración física y las pruebas complementarias que suele precisar el manejo quirúrgico.

Las especialidades a las que se realizaron más derivaciones fueron traumatología, oftalmología, ginecología, dermatología y otorrinolaringología coincidiendo básicamente con los resultados obtenidos en estudios previos $3,11-13,19-20$. Esta tendencia parece relacionarse con la carencia de recursos en los CS 


\begin{tabular}{|c|c|c|c|c|}
\hline \multirow{2}{*}{$\begin{array}{l}\text { Especialidad } \\
\text { Alergología }\end{array}$} & \multirow{2}{*}{$\begin{array}{c}\text { OR } \\
9,962\end{array}$} & \multicolumn{2}{|c|}{ IC 95,0\% para } & \multirow{2}{*}{$\begin{array}{c}\mathbf{p} \\
<0,001\end{array}$} \\
\hline & & 2,808 & 35,340 & \\
\hline Cirugía general & 0,381 & 0,222 & 0,654 & $<0,001$ \\
\hline Digestivo & 0,226 & 0,143 & 0,357 & $<0,001$ \\
\hline Neumología & 2,533 & 1,458 & 4,401 & 0,001 \\
\hline ORL & 7,580 & 1,617 & 35,521 & 0,01 \\
\hline Salud mental & 3,516 & 1,431 & 8,634 & 0,006 \\
\hline Rehabilitación & 4,520 & 2,575 & 7,932 & $<0,001$ \\
\hline Urología & 3,055 & 1,306 & 7,146 & 0,01 \\
\hline
\end{tabular}

Tabla 4. Variables finales predictoras de calidad de la hoja de derivación DEFICIENTE mediante un análisis de regresión logística condicional hacia atrás controlando por sexo, edad del paciente y médico que realiza la hoja de derivación. OR: Odd Ratio; IC: intervalo de confianza; ORL: otorrinolaringología.

para resolver muchos de los motivos de consulta derivados a especialidades médico-quirúrgicas.

Quizás lo más llamativo sea obtener significación estadística en la variable calidad de la HD entre médicos con formación MIR y sin ella y en las características del médico (sexo y ser o no tutor), diferencias no objetivadas en otros estudios similares ${ }^{8-9,20}$. Estos resultados hay que interpretarlos con cautela, no sólo porque nuestra muestra de médicos sea insuficiente para inferir causalidad sino porque la variable del residente como factor de confusión no se ha tenido en cuenta.

Por todo ello, podemos concluir que la introducción de los programas informáticos en las consultas de AP nos proporciona un nuevo formato de valoración de la calidad de la HD, pero falta el desarrollo de criterios de calidad de las mismas. Este trabajo introduce el mundo informático en la gestión de la calidad de la actividad asistencial del médico de AP, proporcionando una nueva metodología para medir la calidad de las HD. También para poder desarrollar intervenciones dirigidas a conocer si aumenta la calidad de las HD y si ésta interviene en la reducción o aumento de las TD.

\section{AGRADECIMIENTOS}

Alfonso Abaigar Martínez de Salinas, Técnico de Salud Pública de la Gerencia de Atención Primaria de Cuenca.

Fernando Salcedo Aguilar, Coordinador de la Unidad Docente de MFyC de Cuenca.

\section{BIBLIOGRAFÍA}

1. Rowlands G, Willis S, Singlenton A. Referrals and relationships: in-practice referrals meetings in a general practice. Fam Pract. 2001; 18:399-406.

2. Forrets $C B$. Primary care gatekeeping and referrals: effective filter or failed experiment? BMJ. 2003; 326:692-5.

3. Rubio Arribas V, Rodríguez Ibáñez ML, Sampedro Martínez E, Victores Benavente C, Alechiguerra García A, Barrio Gamarra JL.Evaluación de la calidad de comunicación entre niveles asistenciales mediante el documento interconsulta. Aten Primaria. 2000; 26:681-4.

4. Forrest CB, Nutting P, Werner JJ, Starfield B, von Schrader S, Rohde C. Managed health plan effects on the specialty referral process: results from the Ambulatory Sentinel Practice Network referral study. Med Care. 2003; 41:242-53.

5. Fustero MV, Garcia-Mata JR, Junod B, Bárcena M ¿Funciona el flujo de información entre los niveles primario y especializado? Análisis de la continuidad asistencial en un Área de Salud. Rev Calidad Asistencial 2001; 16:247-252.

6. Prado Prieto L, García Olmos L, Rodríguez Salvanés F, Otero Puime A. Evaluación de la demanda derivada en atención primaria. Aten Primaria. 2005; 35:146-51.

7. Alonso Pérez de Ágreda JP, Febrel Fordejé M, Huelin Domeco de Jarauta J. Factores asociados a la derivación inadecuada entre atención primaria y especializada: estudio cualitativo en médicos de atención primaria. Gac Sanit. 2000; 14:122-30.

8. Gérvas J, García Olmos LM, Simó J, Peiró S. Paradojas en la derivación de primaria a especializada. Aten Primaria. 2008; 40:253-5.

9. Gérvas J, Starfield B, Violán C, Minué S. GPs with special interests:unanswered questions. Br J Gen Pract. 2007; 57:912-7. 
10. Servicio de Salud de Castilla-La Mancha. Avances tecnológicos. Disponible en : http://sescam.jccm.es/web1/home. do?main=/ciudadanos/avancesMedTecn/turriano.htm1

11. Buitrago F. Relación entre niveles asistenciales. Oviedo: SemFYC; 1995.

12. Morera J, Custodi J, Sánchez L, Miaja F. Análisis de la calidad de la información transmitida entre atención primaria y atención especializada. Medfam. 1991; 3:132-40.

13. Yagüe Hernando AJ, Blanco Ramos MT, Juez Juez AA. Análisis de las derivaciones a las consultas de especialistas realizadas en un centro de salud. Aten Primaria. 1991; 8:472-6.

14. Gómez-Calcerrada D, Pérez Flores D, Marset Campos P. La cita previa, perfil del consultante y accesibilidad. Aten Primaria. 1996; 17:288-91.

15. Huertas Zarco I, Pereiró Berenguer I, Sanfélix Genovés J, Rodríguez Moya R. Mejora de la calidad de la hoja de interconsulta a través de la información. Aten Primaria. 1996; 17:317-20.

16. Irazábal Olabarrieta L, Gutiérrez Ruiz B ¿Funciona la comunicación entre los niveles primario y secundario? Aten Primaria. 1996; 17:376-81.
17. Mimbela Sánchez MM, Foradada Baldellou S. Análisis de la interrelación atención primaria-atención especializada en la derivación de pacientes. Aten Primaria. 1993; 12:65-8.

18. Llobera Canaves J. La derivación de pacientes de la atención primaria a la especializada. Gac Sanit. 1988; 9:271-5.

19. Roset Monrós P, Hernando López T, Lobón Agúndez MC, Rodríguez Fernández C, Sabugal Rodelgo G,Martín Álvarez R, Velázquez García A ¿Existen diferencias en la calidad de los partes de interconsulta emitidos por los dos modelos asistenciales de Atención Primarias (Equipo de Atención Primaria/Modelo tradicional)? Semergen. 1999; 25(3):195-97.

20. Rodríguez Alcalá FJ, Chacón Fuertes J, Esteban Tudela M, Valles Fernández N, López de Castro F, Sánchez Ramiro A. Motivos de interconsulta entre atención primaria y el segundo nivel. Aten Primaria. 2005; 36:137-43.

21. Alberdi-Ordiozola JC, Sáenz-Bajo N. Factores determinantes de la derivación de atención primaria a las consultas externas de atención especializada en la Comunidad de Madrid. Aten Primaria. 2006; 5:253-7. 